\title{
On the Use of Shift Dynamics in Synthesizing Fractal Images
}

\author{
Jonas VALANTINAS, Tomas ŽUMBAKIS \\ Department of Applied Mathematics, Kaunas University of Technology \\ Studentu 50, 3031 Kaunas, Lithuania \\ e-mail:valanti@if.ktu.lt
}

Received: February 2004

\begin{abstract}
The contribution of this paper is a new version of the escape time algorithm adapted to synthesize fractal images, identified with attractors of iterated function systems (IFS). The proposed synthesis algorithm is based on the use of shift dynamics, associated with one or another IFS. The novelty of the algorithm is grounded on two factors. Firstly, the strategy for the separation of extended domains of the inverse affine transformations, specified by IFS, is developed. Secondly, the variable escape time for different points of a synthesized fractal image (IFS attractor) is proposed and explored. Experimental results show that the above two factors ensure uniform rendering of image points (pixels) in colour.
\end{abstract}

Key words: iterated function systems, shift dynamics, escape time algorithm, synthesizing fractal images.

\section{Introduction}

Throughout the last decade development and implementation of fractal image processing technologies was an area of increasing interest. Much was done in preparing and maturing diversified fractal image encoding (compression) ideas (Jacquin, 1992; Fisher, 1994; Wohlberg and Jager, 1999; Valantinas et al., 2002). Constant attention was paid to fractal modelling, to the analysis and synthesis of fractal images (Turner et al., 1998; Peitgen et al., 1992). Elements of fractal geometry (fractal dimension, fractal interpolation, fractal calculus) were put to work in real-world applications (Barnsley, 1993; Evans, 1997; Yokoya et al., 1989).

In the field of computerized real-world image models (digital images) the fractal approach is of outmost importance, because it facilitates perception and understanding of the information content of an image. To say more, it provides us with a powerful means to catch sight of a fundamental real world image property generally known as self-similarity. Due to this property, the research and development of algorithms ("fractal techniques") to extract important fractal parameters from appropriate digital data has received significant attention in recent years. Most rapidly developing areas (let us emphasize it once again) are the use of fractal geometry for synthesizing images, for recognizing patterns in images and performing image data compression. Moreover, the latter areas are closely 
intertwined. Say, the problem of synthesizing (fractal) images is far from being an end in itself. Well-known fractal image compression technologies (Barnsley and Hurd, 1993; Fisher, 1994), without exception, employ fractal image synthesis procedures at the image decoding stage. From this point of view, any attempts to develop, to modify and to put into practice one or another fractal image processing (synthesizing) technique or idea are worthy of great praise.

In this paper, the basic concepts and ideas that are needed to describe, state and solve the problem of synthesizing fractal images, identified with the attractors of iterated function systems (IFS), are introduced and explored (Section 2). In the centre of attention - the shift dynamical system, which generates fractal images (attractors of IFS). A new original approach (idea), leading to practical implementation of the above dynamical system, is proposed (Sections 3, 4 and 5). A number of experimental results are given (Section 6).

\section{Creating Fractal Images - Attractors of IFS}

Let $\left(\mathrm{R}^{2}, d\right)$ be a Euclidean metric space and $\left(H\left(\mathrm{R}^{2}\right), h\right)$ denote the corresponding (fractal) space of nonempty closed subsets, with the Hausdorff distance (metric) $h=$ $\max \{d(A, B), d(B, A)\}$, where

$$
d(A, B)=\max _{P \in A}\{d(P, B)\}, \quad d(P, B)=\min _{Q \in B}\{d(P, Q)\},
$$

for all $A, B \in H\left(\mathrm{R}^{2}\right) ; d(B, A)$ is defined similarly.

Let $\omega_{i}: \mathrm{R}^{2} \rightarrow \mathrm{R}^{2}, i=1,2, \ldots, N$, be a contractive affine transformation on the metric space $\left(\mathrm{R}^{2}, d\right)$, i.e., $\forall P(x, y) \in \mathrm{R}^{2}: \omega_{i}(P)=\omega_{i}(x, y)=\left(a_{i} x+b_{i} y+e_{i}, c_{i} x+\right.$ $\left.d_{i} y+f_{i}\right)$, where $a_{i}, b_{i}, c_{i}, d_{i}, e_{i}, f_{i} \in R$, and let

$$
s_{i}=\sup \left\{\hat{s}_{i} \mid d\left(\omega_{i}(P), \omega_{i}(Q)\right) \leqslant \hat{s}_{i} \cdot d(P, Q), P, Q \in R^{2}\right\}
$$

indicate the (global) contractivity factor for $\omega_{i} ; 0 \leqslant s_{i}<1, i=1,2, \ldots, N$. In what follows, the metric space $\left(\mathrm{R}^{2}, d\right)$ together with a finite set of contractive affine transformations $\omega_{i}: \mathrm{R}^{2} \rightarrow \mathrm{R}^{2}, i=1,2, \ldots, N$, is called an iterated function system (IFS) and is denoted by $\operatorname{IFS}\left\{\mathrm{R}^{2} ; \omega_{1}, \omega_{2}, \ldots, \omega_{N}\right\}$.

Let us move the above affine transformations into the space $\left(H\left(\mathrm{R}^{2}\right), h\right)$. Then $\omega_{i}: H\left(\mathrm{R}^{2}\right) \rightarrow H\left(\mathrm{R}^{2}\right)$, defined by $\omega_{i}(B)=\left\{\omega_{i}(Q) \mid Q \in B\right\}, \forall B \in H\left(\mathrm{R}^{2}\right)$, is a contractive affine transformation on $\left(H\left(\mathrm{R}^{2}\right), h\right)$, with contractivity factor $s_{i}, i=1,2, \ldots, N$.

Combining $\omega_{i}: H\left(\mathrm{R}^{2}\right) \rightarrow H\left(\mathrm{R}^{2}\right), i=1,2, \ldots, N$, we produce a new transformation $W: H\left(\mathrm{R}^{2}\right) \rightarrow H\left(\mathrm{R}^{2}\right)$ on the fractal space $\left(H\left(\mathrm{R}^{2}\right), h\right)$, namely:

$$
W(B)=\omega_{1}(B) \cup \omega_{2}(B) \cup \ldots \cup \omega_{N}(B)=\bigcup_{i=1}^{N} \omega_{i}(B),
$$

for all $B \in H\left(\mathrm{R}^{2}\right)$. We note here that the latter transformation is also contractive, i.e., $h(W(B), W(C)) \leqslant s \cdot h(B, C)$, for all $B, C \in H\left(\mathrm{R}^{2}\right)$; here $s=\max \left\{s_{1}, s_{2}, \ldots, s_{N}\right\}$. 
The only fixed point $A \in H\left(\mathrm{R}^{2}\right)$ of $W$, such that

$$
\begin{aligned}
A & =W(A)=\bigcup_{i=1}^{N} \omega_{i}(A)=\lim _{n \rightarrow \infty}(W \circ W \circ \ldots \circ W)(B) \\
& =\lim _{n \rightarrow \infty}\left(W^{0 n}\right)(B), \quad \forall B \in H\left(\mathrm{R}^{2}\right),
\end{aligned}
$$

represents an attractor (fractal image, fractal) of the IFS (Barnsley, 1993).

Among the algorithms, applied to creating attractors of IFS, we find the following ones - the photocopy (deterministic) algorithm, the random iteration algorithm and the escape time algorithm. The former two algorithms, roughly speaking, are based on the definition of the attractor of an IFS, i.e., explore the fact that the set (attractor, fractal image) $A$ is an attractive fixed point for $W: H\left(\mathrm{R}^{2}\right) \rightarrow H\left(\mathrm{R}^{2}\right)$. These algorithms are reasonably fast and can be used to create sufficiently realistic images.

The third (escape time) algorithm is of particular interest. Usually, it is applied to the analysis of nonlinear mappings in a complex plane $\mathrm{C}$, i.e., to the study of dynamical systems $\{\mathrm{C} ; f\}$ and their orbits, where $f: \mathrm{C} \rightarrow \mathrm{C}$ is a single non-contractive transformation. It appears that such dynamical systems are sources of fractal images (Peitgen et al., 1986). For a special class of fractal images - IFS attractors - no version of the escape time algorithm is adapted in full. Though, the application scheme of the algorithm is well defined and completely perceptible. We here present a brief description of the escape time algorithm, oriented to create (synthesize) fractal images, identified with the attractors of IFS:

1. Let an iterated function system $\operatorname{IFS}\left\{\mathrm{R}^{2} ; \omega_{1}, \omega_{2}, \ldots, \omega_{N}\right\}$, with an attractor $A=$ $\omega_{1}(A) \cup \omega_{2}(A) \cup \ldots \cup \omega_{N}(A) \in H\left(\mathrm{R}^{2}\right)$, be given. Assume that all affine transformations $\omega_{i}: A \rightarrow A(i=1,2, \ldots, N)$ are invertible.

2. Let $\{A ; S\}$ be a shift dynamical system associated with the IFS, where the shift transformation $S: A \rightarrow A$ is defined (for all $a \in A$ ) as follows: $S(a)=\omega_{i}^{-1}(a)$, if $a \in \omega_{i}(A)(i \in\{1,2, \ldots, N\})$ and $a \notin \bigcup_{j(j \neq i)} \omega_{j}(A)$; we say that the point $a$ is in the domain (under the action) of $\omega_{i}^{-1} ; S(a)=\omega_{i_{t}}^{-1}(a)$, if $a \in \omega_{i_{1}}(A) \cap \omega_{i_{2}}(A) \cap$ $\ldots \cap \omega_{i_{r}}(A), i_{t} \in\{1,2, \ldots, N\}, t=1,2, \ldots, r$; we say that the point $a$ is under the action of anyone of transformations $\omega_{i_{t}}^{-1}$.

3. The shift dynamical system $\{A ; S\}$, associated with the given IFS, is extended to $\mathrm{R}^{2}$, i.e., a new dynamical system (an extension) $\left\{\mathrm{R}^{2} ; \widehat{S}\right\}$ is formed. The new shift transformation $\widehat{S}: \mathrm{R}^{2} \rightarrow \mathrm{R}^{2}$ is defined by the equality $\widehat{S}(P)=\omega_{i}^{-1}(P)$, provided the point $P \in \mathrm{R}^{2}$ is under the action of the inverse affine transformation $\omega_{i}^{-1}(i \in\{1,2, \ldots, N\})$. In particular, $\widehat{S}$ maps $A$ onto itself, i.e., $\widehat{S}$ coincides with the transformation $S$, for all points lying on the attractor $A \subset \mathrm{R}^{2}$ (Fig. 1).

4. Let the set (attractor) $A$ fall into a rectangle (viewing window) $\Re$, i.e., $A \subset \Re \subset$ $\mathrm{R}^{2}$. Then, for every point $P \in \Re$, an orbit $\left\{\widehat{S}^{0 n}(P)\right\}_{n=1}^{\Im}$ is calculated numerically; here $\Im$ is a priori defined number of iterations.

5. Let $R$ be a positive number such that the ball $\mathrm{O}$, centred at the symmetry centre $O$ of the viewing window $\Re$, contains both $A$ and $\Re$ (Fig. 1). If, now, $d\left(\widehat{S}^{0 \Im}(P), O\right) \leqslant R$, 
i.e., the orbit of $P \in \Re$ does not escape the ball (after $\Im$ iterations), a conclusion is made - the point $P$ belongs to the attractor $A$ ( $P$ is rendered in colour); otherwise $\left(d\left(\widehat{S}^{0 \Im}(P), O\right)>R\right), P \notin A$.

As it can be seen, the escape time algorithm explores the fact that the set $A$ (attractor of the IFS, fractal image) is a repulsive fixed point for the transformation $\widehat{S}: H\left(\mathrm{R}^{2}\right) \rightarrow$ $H\left(\mathrm{R}^{2}\right)$. Evidently, the orbits of points that lie close to the attractor $A$ take longer to escape from the ball $\mathrm{O}$ than those of points which lie further away.

The main obstacle preventing the escape time algorithm from being adapted to synthesizing of fractal images, associated with IFS, is the following one - neither theoretical nor empirical criteria exist for the separation of extended domains of the inverse affine transformations. Some authors mention in passing that the extensions of the domains can be defined with the aid of straight lines (Barnsley, 1993). Unfortunately, in a general case, no appreciable results have been obtained and published till now.

So, broadly speaking, the search for the named above criteria becomes an object of theoretical interest and theoretical investigations despite even the final outcome - practical applicability (in the efficiency sense) of the sought-for criteria themselves.

This paper introduces a new interesting approach (idea) to separating extended domains (in $\mathrm{R}^{2}$ ) of the inverse affine transformations (Section 3). The proposed idea facilitates adaptation of the escape time algorithm to synthesizing of fractal images (attractors of IFS).

Also, to ensure uniform rendering of points (pixels) $P \in \Re$ in colour, a variable number of iterations $\Im=\Im(P)$, for different points in the viewing window $\Re$, is introduced (Section 5).

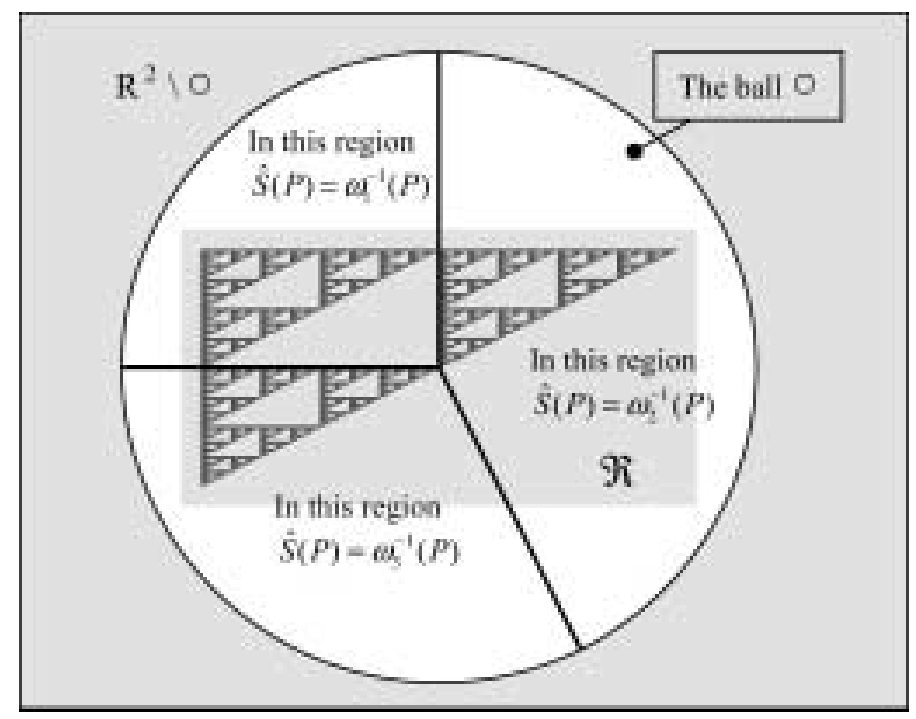

Fig. 1. The dynamical system $\left\{\mathrm{R}^{2} ; \widehat{S}\right\}$ is obtained by extending the definition of the shift dynamical system $\{A ; S\}$, associated with IFS $\left\{\mathrm{R}^{2} ; \omega_{1}, \omega_{2}, \omega_{3}\right\} ; A=\omega_{1}(A) \cup \omega_{2}(A) \cup \omega_{3}(A)$ is the IFS attractor (Sierpinski triangle); bold type lines separate extended domains for affine transformations. 


\section{Determination of Extended Domains for Inverse Affine Transformations - Stating the Problem}

Consider an IFS $\left\{\mathrm{R}^{2} ; \omega_{1}, \omega_{2}, \ldots, \omega_{N}\right\}$, whose attractor is the set $A \in H\left(\mathrm{R}^{2}\right)$. Let us denote the fixed point and the (global) contractivity factor of $\omega_{i}(i \in\{1,2, \ldots, N\})$ by $M_{i}$ and $s_{i}$, respectively. For more detailed description of the "behaviour" of $\omega_{i}(i \in$ $\{1,2, \ldots, N\})$ in $\left(\mathrm{R}^{2}, d\right)$, it is expedient to introduce a local contractivity factor $s_{i}(P)$, $P \in \mathrm{R}^{2}$, acting in the direction, specified by the vector $\overrightarrow{P M_{i}}$, namely:

$$
s_{i}(P)=\sqrt{\left(a_{i} \cos \varphi_{i}+b_{i} \sin \varphi_{i}\right)^{2}+\left(c_{i} \cos \varphi_{i}+d_{i} \sin \varphi_{i}\right)^{2}}
$$

here: $a_{i}, b_{i}, c_{i}, d_{i}$ are parameters (real numbers) of $\omega_{i} ; \varphi_{i}=\varphi_{i}(P)$ is the angle between $\overrightarrow{P M_{i}}$ and the positive direction of the axis of abscissae. More convenient formula, especially for practical applications, is the following one:

$$
s_{i}(P)=d\left(\omega_{i}(P), M_{i}\right) / d\left(P, M_{i}\right)
$$

Suppose, now, that $\left\{\mathrm{R}^{2} ; \widehat{S}\right\}$ is an extension of the shift dynamical system $\{A ; S\}$, associated with the IFS. As it was mentioned above (Section 2), the shift transformation $\widehat{S}$ maps any point $P \in \mathrm{R}^{2}$ into a new point $P_{i}=\widehat{S}(P)=\omega_{i}^{-1}(P)$, provided $P$ is under the action of $\omega_{i}^{-1}(i \in\{1,2, \ldots, N\})$.

In a theoretical sense, for the separation of extended domains of the inverse affine transformations, the use can be made of the following criterion

$$
\min _{i \in\{1,2, \ldots, N\}}\left\{d\left(P, \omega_{i}(A)\right) / s_{i}(P)\right\}=\frac{1}{s_{i^{\circ}}(P)} d\left(P, \omega_{i^{\circ}}(A)\right)
$$

(index $i^{\circ}$ shows an affine transformation, into whose domain the point $P$ falls in). Unfortunately, practical implementation of the criterion (expression (2)) is complicated, because neither the attractor $A$ nor its copies $\omega_{i}(A), i \in\{1,2, \ldots, N\}$, are known.

In this context, we are to formalize the application scheme of the escape time algorithm, described in Section 2. Firstly, let us construct a set $I(\Im)$ of ordered $\Im$-tuples ( $\Im$ is a positive integer), i.e.,

$$
I(\Im)=\left\{\left(i_{1}, i_{2}, \ldots, i_{\Im}\right) \mid i_{k} \in\{1,2, \ldots, N\}, k=1,2, \ldots, \Im\right\}
$$

Clearly, the number of elements in $I(\Im)$ equals $N^{\Im}$.

Now, for a particular $\Im$-tuple (ordered collection of indices) $\left(i_{1}, i_{2}, \ldots, i_{\Im}\right)$ and for any point $P \in \Re \subset \mathrm{R}^{2}$, we can compute an orbit (sequence), comprising $\Im$ points, namely: $\left\{P_{i_{1}}, P_{i_{1}, i_{2}}, \ldots, P_{i_{1}, i_{2}, \ldots, i_{\Im}}\right\}$, where

$$
P_{i_{1}, i_{2}, \ldots, i_{k}}=\left(\omega_{i_{k}}^{-1} \circ \ldots \circ \omega_{i_{2}}^{-1} \circ \omega_{i_{1}}^{-1}\right)(P),
$$

for all $k \in\{1,2, \ldots, \Im\}$. 
By choosing properly both the radius $R$ of the ball $\mathrm{O}$ and the number of iterations $\Im$, we come to the conclusion (based on the material in Section 2$)$ - the point $P(P \in \Re)$ belongs to the attractor $A$ if and only if the condition

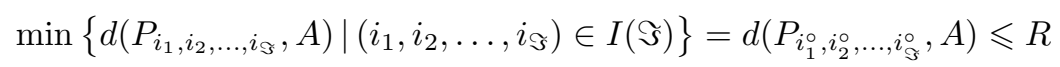

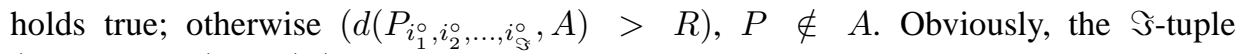
$\left(i_{1}^{\circ}, i_{2}^{\circ}, \ldots, i_{\Im}^{\circ}\right) \in I(\Im)$ indicates the case when extended domains are defined correctly for all points in $\left\{\widehat{S}^{0 n}(P)\right\}_{n=1}^{\Im}$.

Mainly, on account of the inequality $\operatorname{diam}(A) \ll R$, condition (3) can be reformulated this way:

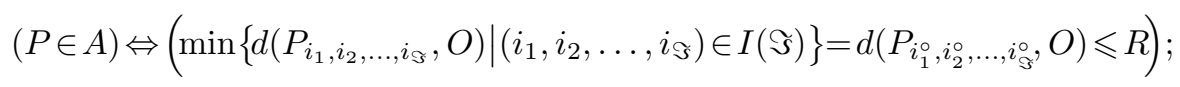

here $O$ is the symmetry centre of the viewing window $\Re$.

The direct implementation of the above formal approach (criterion (4)) is problematic, since cardinality of the set $I(\Im)$ increases drastically, with the number of iterations $\Im$ increasing.

\section{The New Strategy for Determination of Extended Domains - Solving the Problem}

We here present a new strategy (criterion) for the separation of extended domains (in the Euclidean space $\left.\left(\mathrm{R}^{2}, d\right)\right)$ of the inverse affine transformations, associated with an $\operatorname{IFS}\left\{\mathrm{R}^{2} ; \omega_{1}, \omega_{2}, \ldots, \omega_{N}\right\}$. It leads to practical implementation of the condition (4) (Section 3). The essence of the proposed criterion - the point $P_{i_{1}^{\circ}, i_{2}^{\circ}, \ldots, i_{k}^{\circ}}(k \in\{1,2, \ldots, \Im-$ $1\}$ ) of a sequentially computed orbit $\left\{\widehat{S}^{0 n}(P)\right\}_{n=1}^{\Im}, P \in \Re$, is under the action of the inverse affine transformation $\omega_{i_{k+1}^{\circ}}^{-1}\left(i_{k+1}^{\circ} \in\{1,2, \ldots, N\}\right)$ if and only if:

$$
\begin{aligned}
& \min _{i_{k+1}, \ldots, i_{k+\tau} \in\{1,2, \ldots, N\}}\left\{d\left(P_{i_{1}^{\circ}, \ldots, i_{k}^{\circ}, i_{k+1}, \ldots, i_{k+\tau}}, P_{i_{1}^{\circ}, \ldots, i_{k}^{\circ}}\right)\right\} \\
& =\min _{i_{k+2}, \ldots, i_{k+\tau} \in\{1,2, \ldots, N\}}\left\{d\left(P_{i_{1}^{\circ}, \ldots, i_{k+1}^{\circ}, i_{k+2}, \ldots, i_{k+\tau}}, P_{i_{1}^{\circ}, \ldots, i_{k}^{\circ}}\right)\right\} \quad(2 \leqslant \tau \leqslant N) .
\end{aligned}
$$

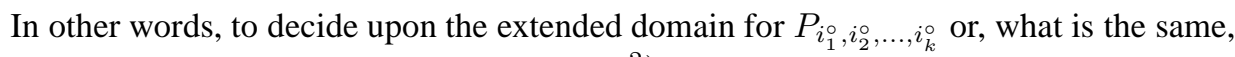
for the separation of extended domains (in $\mathrm{R}^{2}$ ), the strategy " $\tau$ steps ahead" is proposed. The strategy (criterion) is based on the following factors (easy to prove):

- if $P\left(P \in \mathrm{R}^{2}\right)$ is in the domain of the inverse affine transformation $\omega_{i}^{-1}(i \in$ $\{1,2, \ldots, N\})$, then

$$
d\left(\omega_{i}^{-1}(P), A\right) \leqslant d\left(\omega_{j}^{-1}(P), A\right),
$$

for all $j \in\{1,2, \ldots, N\}, j \neq i$; this assertion follows directly from the expression (2) (Section 3); 
- for any point $P \in \mathrm{R}^{2}$ and its image $P_{i}=\omega_{i}^{-1}(P)(i \in\{1,2, \ldots, N\})$ the inequality

$$
d\left(P_{i}, P\right) \geqslant d(P, A) \cdot(1-s(P)) / s(P) \geqslant d(P, A) \cdot(1-s) / s
$$

is true; $s(P)=\max \left\{s_{j}(P) \mid j=1,2, \ldots, N\right\}$. It means that the distance between two points, $P$ and $P_{i}$, increases, as $P$ moves away from the attractor $A$;

- the relationship

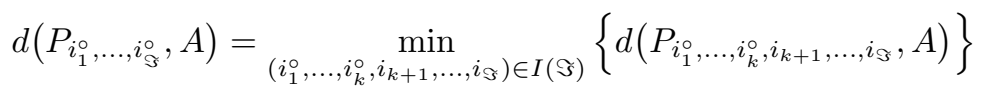

is valid (follows from (3)), for all values of $k(k \in\{1,2, \ldots, \Im-1\})$. Consequently, the orbit $\left\{\widehat{S}^{0 n}(P)\right\}_{n=1}^{\Im}$ of the point $P \in \Re$ can be calculated in consecutive order.

The obvious advantage of the proposed criterion (expression (5)) - only $\tau(\tau \ll \Im)$ steps ahead are needed to decide upon the correctness of the choice of a particular ex-

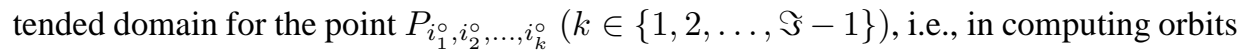
for points from the viewing window $\Re$, we avoid complete analysis of $\Im$-tuples in the set $I(\Im)$ (expression (4); Section 3). The latter analysis is replaced with the detailed investigation (at every orbit point) of $\tau$-tuples $\left(i_{1}, i_{2}, \ldots, i_{\tau}\right) \in I(\tau)$, where $|I(\tau)|=N^{\tau}$, and $\Im \cdot N^{\tau}$ is much less than $|I(\Im)|=N^{\Im}$. At the same time, the criterion (strategy) is comparatively simple and easy to use.

Below, we present an illustrative example where the proposed strategy " $\tau$ steps ahead" is employed (Figs. 2, 3). We treat an IFS $\left\{\mathrm{R}^{2} ; \omega_{1}, \omega_{2}\right\}$, where $\omega_{1}=\omega_{1}(x, y)=$ $(-1 / \sqrt{2} y+100,1 / \sqrt{2} x)$ and $\omega_{2}=\omega_{2}(x, y)=(-1 / \sqrt{2} y+200,1 / \sqrt{2} x)$. The IFS attractor $A=\omega_{1}(A) \cup \omega_{2}(A)=\{(x, y) \mid 0 \leqslant x \leqslant 200,0 \leqslant y \leqslant 100 \sqrt{2}\}$ is the union of two copies of itself, each scaled by a factor $1 / \sqrt{2}$. The inverse transformations are $\omega_{1}^{-1}=$ $\omega_{1}^{-1}(x, y)=(\sqrt{2} y,-\sqrt{2} x+100 \sqrt{2})$ and $\omega_{2}^{-1}=\omega_{2}^{-1}(x, y)=(\sqrt{2} y,-\sqrt{2} x+200 \sqrt{2})$.

The dynamical system $\left\{\mathrm{R}^{2} ; \widehat{S}\right\}$, defined by

$$
\widehat{S}(P)=\widehat{S}(x, y)= \begin{cases}\omega_{1}^{-1}(x, y), & x>100, \\ \omega_{2}^{-1}(x, y), & x \leqslant 100\end{cases}
$$

is an extension of the shift dynamical system $\{A ; S\}$, associated with the given IFS. The latter assertion is based on the task-oriented analysis of points (and their orbits) from the viewing window $\Re$ (Fig. 2).

For instance, let us take a point $P(208,100 \sqrt{2}) \in \Re$ and construct its orbit $\left\{\widehat{S}^{0 n}(P)\right\}_{n=1}^{\Im}$, using the strategy " 2 steps ahead". Firstly, the point $P$ itself falls into the extended domain of the inverse affine transformation $\omega_{2}^{-1}$, since (criterion (5))

$$
\min _{i_{1}, i_{2} \in\{1,2\}}\left\{d\left(P_{i_{1}, i_{2}}, P\right)\right\}=\min _{i_{2} \in\{1,2\}}\left\{d\left(P_{2, i_{2}}, P\right)\right\} ;
$$




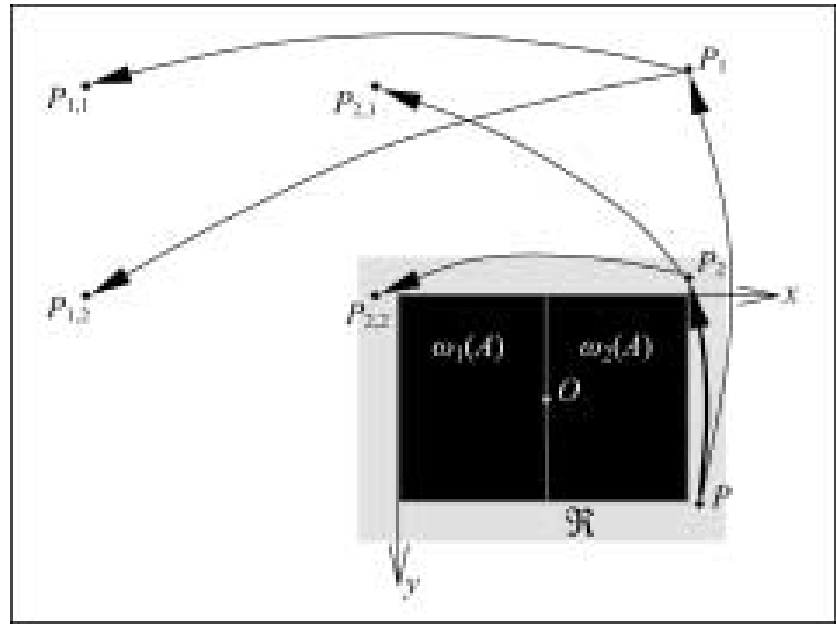

(a)

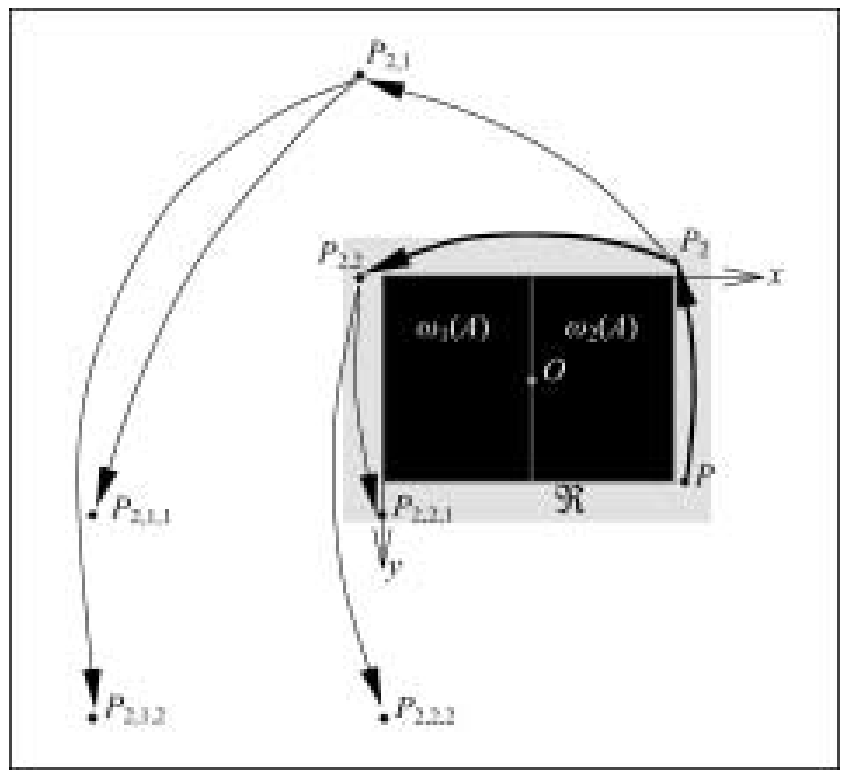

(b)

Fig. 2. Application of the strategy "2 steps ahead": (a) processing of the initial point $P \in \Re$; (b) processing of the point $P_{2}=\omega_{2}^{-1}(P)$.

here $P_{i, j}=\left(\omega_{j}^{-1} \circ \omega_{i}^{-1}\right)(P)$, for all $i, j \in\{1,2\}$; thus, $i_{1}^{\circ}=2$ (Fig. 2a). Secondly, for the newly obtained orbit point $P_{2}=P_{2}(200,-8 \sqrt{2})=\omega_{2}^{-1}(P)$, application of the strategy " 2 steps ahead" leads to the following result

$$
\min _{i_{2}, i_{3} \in\{1,2\}}\left\{d\left(P_{2, i_{2}, i_{3}}, P_{2}\right)\right\}=\min _{i_{3} \in\{1,2\}}\left\{d\left(P_{2,2, i_{3}}, P_{2}\right)\right\}
$$


consequently, the point $P_{2}$ is under the action of $\omega_{2}^{-1}$, i.e., $i_{2}^{\circ}=2$ (Fig. 2b). In the same way, it can be shown that the point $P_{2,2}=\omega_{2}^{-1}\left(P_{2}\right)$ falls into the domain of $\omega_{1}^{-1}$, $P_{2,2,1}=\omega_{1}^{-1}\left(P_{2,2}\right)-$ into the domain of $\omega_{1}^{-1}$, and so on.

Now, if both the radius $R$ of the ball $\mathrm{O}$ and the number of iterations $\Im$ are fixed (say, $R=400, \Im=14)$, then repeated application of the strategy " 2 steps ahead" generates an orbit $\left\{\widehat{S}^{0 n}(P)\right\}_{n=1}^{14}$, which escapes the ball O on the 11-th iteration (Fig. 3). So, the point $P$ doesn't belong to the IFS attractor $A$. Similar reasoning is applicable to all points in $\Re$.

One can easily ascertain that the computational complexity (the number of mappings, performed in evaluating the orbit of $P \in \Re$ ) has been reduced considerably, when compared with the direct implementation of the criterion (4) (Section 3). Really, $\Im \cdot N^{\tau}=14 \cdot 2^{2}=56 \ll N^{\Im}=2^{14}=16384$.

Finally, in order to simplify (optimize) calculations, one can define preliminary all possible superpositions $\omega_{i_{1}, i_{2}, \ldots, i_{\tau}}^{-1}: \mathrm{R}^{2} \rightarrow \mathrm{R}^{2}$, specified by formulae

$$
\omega_{i_{1}, i_{2}, \ldots, i_{\tau}}^{-1}(P)=\left(\omega_{i_{\tau}}^{-1} \circ \ldots \circ \omega_{i_{2}}^{-1} \circ \omega_{i_{1}}^{-1}\right)(P)
$$

$i_{k} \in\{1,2, \ldots, N\}, k=1,2, \ldots, \tau$, and put them to work in deriving criterion values (expression (5)).

It is worth emphasizing, the developed criterion "works" well, provided the dispersion of contractivity factors $s_{i}$ for affine transformations $\omega_{i}, i=1,2, \ldots, N$, is small enough $\left(\left|s_{i}-s_{j}\right| \leqslant \Delta, i=1,2, \ldots, N ; \Delta=0-0.1\right)$. In other cases, to insure higher quality of synthesized fractal images, corrections of the number of iterations, depending on the frequences of the inverse affine transformations (in $\left(i_{1}^{\circ}, i_{2}^{\circ}, \ldots, i_{\Im}^{\circ}\right)$ ), should be made. In

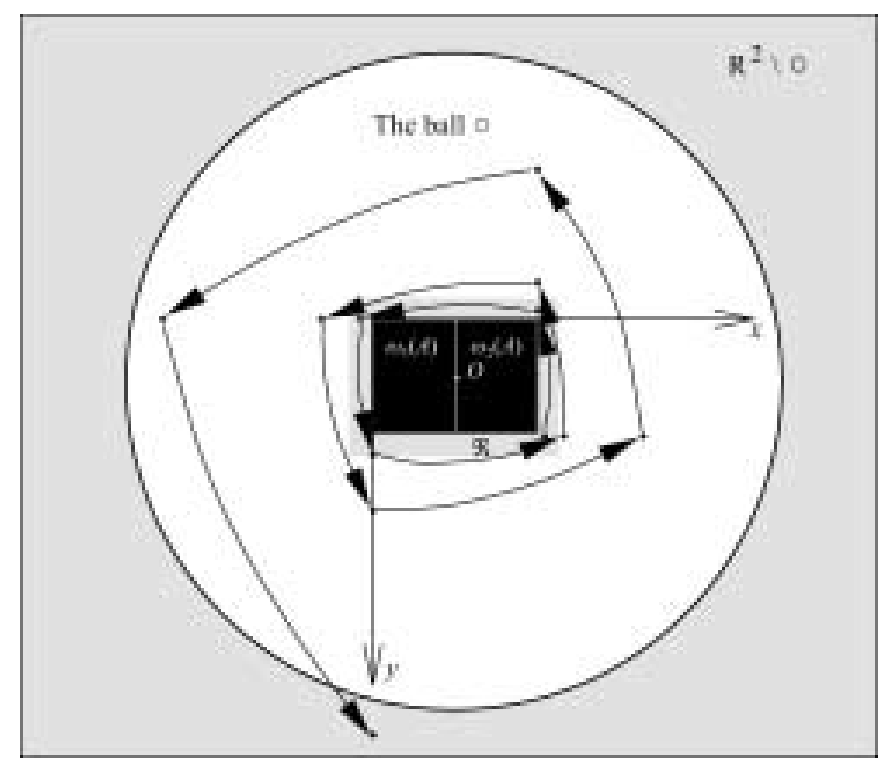

Fig. 3. The orbit $\left\{\widehat{S}^{0 n}(P)\right\}_{n=1}^{14}$ escapes the ball $\mathrm{O}-$ the initial point $P$ doesn't belong to the IFS attractor $A$. 
other words, every point $P \in \Re$ should be associated with its "own" escape time, i.e., with its "own" number of iterations $\Im=\Im(P)$.

\section{Implementation of the Variable Escape Time}

Consider an $\operatorname{IFS}\left\{\mathrm{R}^{2} ; \omega_{1}, \omega_{2}, \ldots, \omega_{N}\right\}$ with contractivity factor $s\left(s=\max \left\{s_{1}, s_{2}, \ldots\right.\right.$, $\left.\left.s_{N}\right\}\right)$. Let $M_{1}, M_{2}, \ldots, M_{N}$ denote fixed points of contractive affine transformations $\omega_{1}, \omega_{2}, \ldots, \omega_{N}$, respectively.

For a single affine transformation $\omega_{i}(i \in\{1,2, \ldots, N\})$, the orbit $\left\{\left(\omega_{i}^{-1}\right)^{0 n}(P)\right\}_{n=1}^{\Im_{i}}$ of the point $P$, such that $d\left(P, M_{i}\right) \geqslant \delta$ ( $\delta$ is a small positive number), escapes the ball with radius $R$ and centre at $M_{i}$ if and only if

$$
\Im_{i} \geqslant \log (R / \delta) / \log \left(1 / s_{i}\right)
$$

To say more, if $s_{i}>s_{j}(i, j \in\{1,2, \ldots, N\})$, then points lying in the neighbourhood of $M_{j}$ escape faster than those lying in the neighbourhood of $M_{i}$.

Based on this understanding, the following procedure, for the determination of the number of iterations for points in a viewing window (and outside it), is proposed:

1. The initial maximal escape time (in the number of iterations) $\Im_{\max }(P)$ for the point $P \in \Re$, which falls into the extended domain of $\omega_{i_{1}^{\circ}}^{-1}\left(i_{1}^{\circ} \in\{1,2, \ldots, N\}\right)$, is found:

$$
\Im_{\max }(P) \geqslant-\log (R / \delta) / \log \mathrm{s}_{i_{1}^{\circ}}
$$

where $R$ is the radius of the ball $\mathrm{O}$, containing both the attractor $A$ of the IFS and the viewing window $\Re ; s_{i_{1}^{\circ}}$ is the (global) contractivity factor for $\omega_{i_{1}^{\circ}}^{-1}$; for practical applications, $\delta=0.1-1.5$.

2. Let $P_{i_{1}^{\circ}, i_{2}^{\circ}, \ldots, i_{k}^{\circ}}$ be the $k$-th point in the orbit of $P \in \Re$, and the number of iterations (escape time) left equal $\Im_{\text {left }}$ (initially, $\Im_{\text {left }}=\Im_{\max }(P)$ ). Now, if $P_{i_{1}^{\circ}, i_{2}^{\circ}, \ldots, i_{k}^{\circ}}$ is under the action of the inverse affine transformation $\omega_{i_{k+1}^{\circ}}^{-1}\left(i_{k+1}^{\circ} \in\{1,2, \ldots, N\}\right)$, then the next orbit point $P_{i_{1}^{\circ}, i_{2}^{\circ}, \ldots, i_{k+1}^{\circ}}$ is computed, and the number $\Im_{l e f t}$ undergoes corrections:

$$
\Im_{l e f t}:=\Im_{l e f t}-\frac{\log s_{i_{k+1}^{\circ}}\left(P_{i_{1}^{\circ}, i_{2}^{\circ}, \ldots, i_{k+1}^{\circ}}\right)}{\log s_{i_{1}^{\circ}}},
$$

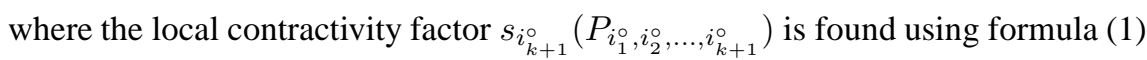
(Section 3), i.e.,

$$
s_{i_{k+1}^{\circ}}\left(P_{i_{1}^{\circ}, i_{2}^{\circ}, \ldots, i_{k+1}^{\circ}}\right)=\frac{d\left(P_{i_{1}^{\circ}, i_{2}^{\circ}, \ldots, i_{k}^{\circ}}, M_{i_{k+1}^{\circ}}\right)}{d\left(P_{i_{1}^{\circ}, i_{2}^{\circ}, \ldots, i_{k+1}^{\circ}}, M_{i_{k+1}^{\circ}}\right)} .
$$

3. If $\Im_{\text {left }}<0$, the condition (4) (Section 3) is verified. Computing of the orbit (for $P$ ) is terminated. If $\Im_{l e f t} \geqslant 0$, then $k:=k+1$ and the move to step 2 is made. 
4. The point $P \in \Re$ is rendered in colour.

Finally, if a given $\operatorname{IFS}\left\{\mathrm{R}^{2} ; \omega_{1}, \omega_{2}, \ldots, \omega_{N}\right\}$ contains only similarity transformations, then their contractivity factors, being invariant with respect to a chosen direction, can be computed once and beforehand. It leads to some time savings in applying the proposed synthesis algorithm (expressions (4)-(7); Sections 3-5).

\section{Experimental Results}

Computer realization (programming language C\#.NET) of both the proposed criterion for the determination of extended domains for inverse affine transformations and the new version of the escape time algorithm, adapted to synthesizing of fractal images, identified with attractors of IFS, was done. Some experimental (synthesis) results are presented below (Figs. 4-6).

Extended domains for the inverse affine transformations, associated with the $\operatorname{IFS}\left\{\mathrm{R}^{2} ; \omega_{1}, \omega_{2}\right\}\left(\omega_{1}: a_{1}=-0.5, b_{1}=-0.5, c_{1}=0.5, d_{1}=-0.5, e_{1}=-48.8\right.$, $\left.f_{1}=8 ; \omega_{2}: a_{2}=0.5, b_{2}=-0.5, c_{2}=0.5, d_{2}=0.5, e_{2}=-86.8, f_{2}=58.4\right)$ and the IFS $\left\{\mathrm{R}^{2} ; \omega_{1}, \omega_{2}, \omega_{3}\right\}\left(\omega_{1}: a_{1}=0.5 ; b_{1}=0, c_{1}=0, d_{1}=0.5, e_{1}=0, f_{1}=0\right.$; $\omega_{2}: a_{2}=0.28, b_{2}=-0.4, c_{2}=0.4, d_{2}=0.28, e_{2}=134, f_{2}=8 ; \omega_{3}: a_{3}=0.5$, $b_{3}=0, c_{3}=0, d_{3}=0.5, e_{3}=8, f_{3}=0$ ), are shown in Fig. 4 (fixed points of the affine transformations are coloured in black). Synthesis results (fractal images, attractors of the

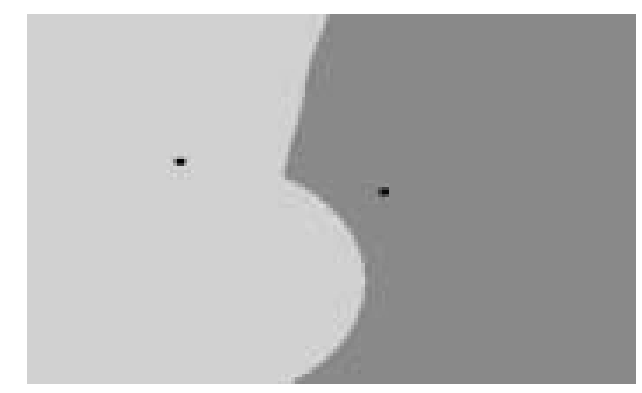

(a)

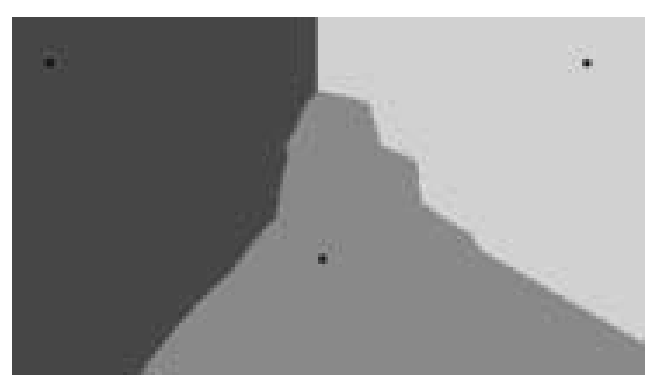

(b)

Fig. 4. Extended domains for the affine transformations: (a) $\operatorname{IFS}\left\{R^{2} ; \omega_{1}, \omega_{2}\right\}$; (b) $\operatorname{IFS}\left\{R^{2} ; \omega_{1}, \omega_{2}, \omega_{3}\right\}$. 


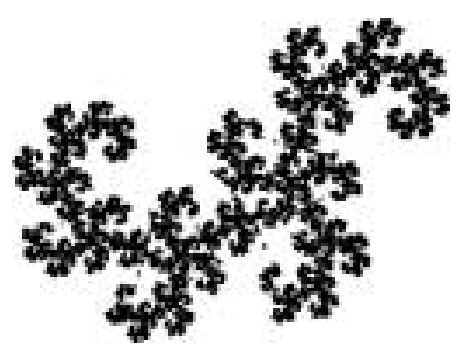

(a)

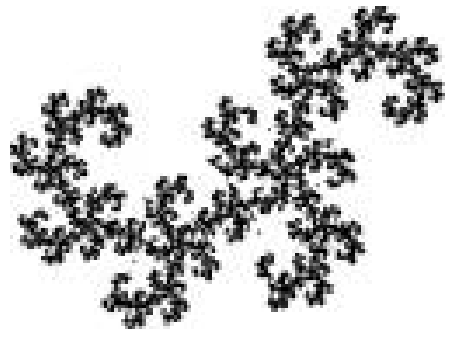

(b)

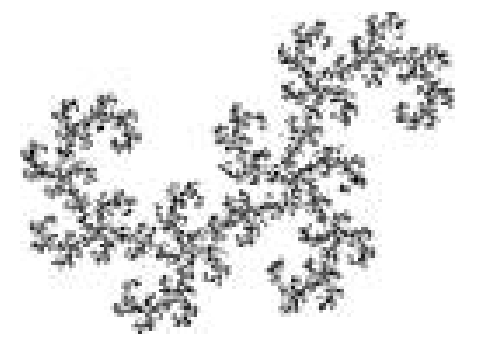

(c)

Fig. 5. Synthesis results $-\operatorname{IFS}\left\{\mathrm{R}^{2} ; \omega_{1}, \omega_{2}\right\}$ : (a) $R=500 ; \delta=1$; (b) $R=500 ; \delta=0.75$;

(c) $R=500 ; \delta=0.15$.

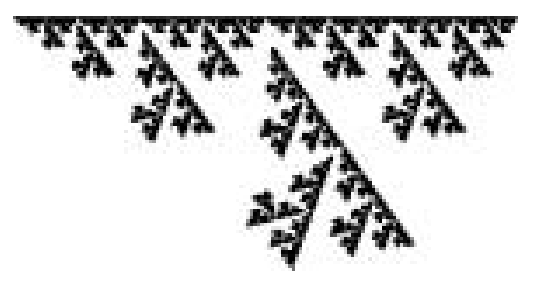

(a)

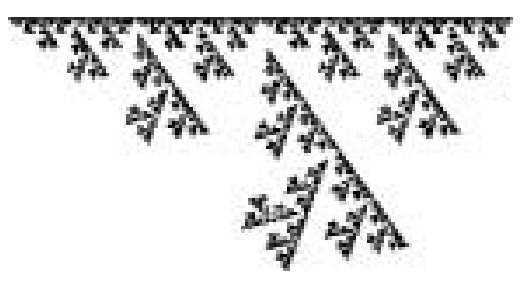

(b)

Fig. 6. Synthesis results $-\operatorname{IFS}\left\{\mathrm{R}^{2} ; \omega_{1}, \omega_{2}, \omega_{3}\right\}$ : (a) $R=700 ; \delta=1$; (b) $R=700 ; \delta=0.4$.

above IFS) are presented in Figs. 5, 6. As it can be seen, the quality of synthesized fractal images highly depends on the initially prescribed number of iterations (escape time) $\Im_{\max }$, which, in its turn, is a function of the parameter $\delta$. To say more, the latter parameter enables us to balance between "poorness" and "coarseness" of the resulting images (Fig. 5).

Computational time of the developed synthesis algorithm depends on the number of affine transformations entering a particular IFS. Preliminary results show that the algorithm is slower, when compared with the random iteration algorithm, but is slightly faster than the deterministic one. 


\section{Conclusion}

Various algorithms for the synthesis of fractal images, identified with the attractors of iterated function systems (IFS) are known, namely: the photocopy (deterministic) algorithm, the random iteration algorithm and the escape time algorithm. The former two algorithms, roughly speaking, are based on the definition of the attractor of an IFS. The third (escape time) algorithm is characterized as being more universal, and, as a rule, is applied to the analysis and visualization of nonlinear mappings, acting in a complex plane. Unfortunately, no version of the latter algorithm is adapted to create fractal images, identified with the attractors of IFS.

The main and serious obstacle in exploring this algorithm - rather complicated and embarrasing determination of extended domains (in a Euclidean space) for the inverse affine transformations, comprising the shift dynamical system, associated with one or another IFS.

In the paper, a new original criterion (strategy) for the separation of the said extended domains is proposed. The criterion creates conditions for the adaptation of the escape time algorithm to the synthesis of attractors (fractal images) of IFS of various types (totally disconnected, just-touching, overlapping). The criterion itself relies upon the sequential realization of the shift dynamical system mentioned above.

The preliminary experimental results show that the new version of the escape time algorithm proved correct. To say more, the obvious theoretical result was gained - the "escape times" idea was fitted to the synthesis of IFS attractors.

In the future, some additional analysis, concerning the quality of synthesized fractal images (in particular, with respect to their fractal dimension) is supposed. In parallels, justified applicability of the developed image synthesis approach to fractal image decoding procedures is planned too.

\section{References}

Arnaud, J. (1992). Image coding based on a fractal theory of iterated contractive image transformations. IEEE Transactions on Image Processing, 1(1), 18-30.

Barnsley, M.F. (1993). Fractals Everywhere. Academic Press Professional, Cambridge.

Barnsley, M.F., and L.P. Hurd (1993). Fractal Image Compression. AK Peters, Wellesley, Mass.

Brent, W., and G. de Jager (1999). A review of the fractal image coding literature. IEEE Transactions on Image Processing, 8(12), 1716-1729.

Evans, A.K. (1997). Fourier dimension, fractal dimension and the fractional derivative. Technical Report 23, SERCentre, De Montfort University, Leicester LE $19 \mathrm{BH}$.

Fisher, Y. (1994). Fractal Image Compression - Theory and Application. Springer-Verlag, New York.

Peitgen, H.-O., and P.H. Richter (1986). The Beauty of Fractals: Images of Complex Dynamical Systems. Springler-Verlag, New York.

Peitgen, H.-O., J. Hartmut, S. Dietmar (1992). Chaos and Fractals. Springer-Verlag.

Turner, M.J., J.M. Blackledge, P.R. Andrews (1998). Fractal Geometry in Digital Imaging. Academic Press, Cambridge.

Valantinas, J., N. Morkevičius, T. Žumbakis (2002). Accelerating compression times in block-based fractal image coding procedures. In Proceedings of the 20th Eurographics UK Conference. Leicester (De Montfort university, UK), IEEE Computer Society Press (Los Alamitos, California). pp. 83-88.

Yokoya, N., F. Yamamoto and N. Funakubo (1989). Fractal based analysis and interpolation of 3d natural surface shapes. Computer Vision Graphics and Image Processing, 46, 284-302. 
J. Valantinas is an associated professor at the Department of Applied Mathematics, Kaunas University of Technology. The area of scientific interest - discrete transforms, digital image processing, fractal technologies.

T. Žumbakis is a doctoral student at the Department of Applied Mathematics, Kaunas University of Technology. The research area - fractal image encoding technologies.

\section{Poslinkiu dinamikos taikymas sintezuojant fraktalinius vaizdus} Jonas VALANTINAS, Tomas ŽUMBAKIS

Šiame straipsnyje pateikiama nauja pabėgimo laiko algoritmo versija, adaptuota sintezuoti fraktaliniams vaizdams, tapatinamiems su iteruotuju funkcijų sistemu (IFS) atraktoriais. Siūlomas sintezès algoritmas remiasi tiksliniu poslinkiu dinamikos, aprašomos konkrečia IFS, taikymu. Algoritmo naujumas grindžiamas dviem faktoriais. Pirma, sudaryta nauja originali IFS sudarančiu afiniuju transformaciju veikimo zonu atskyrimo strategija. Antra, realizuota sintezuojamo fraktalinio vaizdo tašku kintamo pabėgimo laiko idejja. Atlikti eksperimentai rodo, jog abu paminèti faktoriai sąlygoja tolygu spalvinį vaizdo užpildymą. 